Aim of the study: Inactivation of the tumor suppressor E-cadherin ( $\mathrm{CDH} 1)$ and its decreased expression is an important occurrence during carcinogenesis. Nevertheless, the relationship of $\mathrm{CDH} 1$ expression and the promoter methylation with laryngeal cancer cell aggressiveness is still unclear. The purpose of this study was to elucidate the gene and protein E-cadherin expression and the DNA methylation levels and to describe the correlations with morphological features in squamous cell laryngeal cancer.

Material and methods: The authors studied E-cadherin and the DNA methylation level in 86 cases to gain a further understanding of the clinicopathologic significance of analyzed parameters. The pathological evaluation included pTNM classification and the tumor front grading (TFG) criteria. Quantitative analysis of the amplified product in real time (qRT-PCR) for estimation of CDH1 mRNA was used. The methylation status was investigated by using methyl-specific polymerase chain reaction (MSP). The level of $\mathrm{CDH} 1$ protein expression by Western blot was determined.

Results: Downregulation of E-cadherin was found to be related to promoter methylation $(p<0.001)$. In tumors with the highest invasiveness according to TFG criteria the lowest E-cadherin gene and protein level in the study group was observed $(p=0.046$ and $p=0.0002$, respectively). In SCLC with muscle and cartilage invasion and disperse infiltration the lowest $\mathrm{CDH} 1$ gene and protein expression was noted $(p=0.0003$ and $p=0.003$ for deep invasion, $p=0.033$ and $p=0.003$ for multifocal infiltration, respectively).

Conclusions: The current findings suggest an association of E-cadherin tumor expression with progression of laryngeal cancer. $\mathrm{CDH} 1$ gene level may be an auxiliary molecular marker for advanced cases of laryngeal carcinoma; however, further studies are necessary.

Key words: laryngeal carcinoma, E-cadherin $(\mathrm{CDH} 1)$, promoter methylation, mode and depth of invasion.

\section{Diagnostic impact of promoter methylation and E-cadherin gene and protein expression levels in laryngeal carcinoma}

\author{
Katarzyna Starska1, Ewa Forma², Iwona Lewy-Trenda 3 , Paweł Papież ${ }^{4}$ \\ Jan Wośs, Magdalena Bryśs
}

1Department of Otholaryngology and Laryngological Oncology, Medical University of Lodz, Poland

2Department of Cytobiochemistry, University of Lodz, Lodz, Poland

${ }^{3}$ Department of Pathomorphology, Medical University of Lodz, Lodz, Poland

${ }^{4}$ Department of Laryngology, Żeromski Hospital of Krakow, Poland

E-cadherin ( $\mathrm{CDH} 1)$ is a cell adhesion molecule that mediates cell-cell adhesion and thus can modulate tumor invasiveness [1-4]. The E-cadherin gene is located on chromosome 16q22.1 [5]. Mutations and promoter methylation at this site lead to loss of function of this tumor suppressor gene [1, 5]. Many studies indicate that $\mathrm{CDH} 1$ inactivation correlates with decreased diseasefree survival, poor prognosis, and metastasis in various malignancies including breast, thyroid, pancreatic and colorectal cancers [1-6]. E-cadherin is a component of the $\beta$-catenin/cadherin complex, which controls cell-cell adhesion and influences cell migration and in this way acts as an invasion suppressor [7-9]. CDH1 is involved in various cancer pathogenesis, but its role is not clearly defined [2]. In numerous studies the loss of intercellular adhesion by E-cadherin is a fundamental change that occurs during the tumorigenesis and progression of many neoplasms [10-14]. Indeed, the loss of E-cadherin-mediated adhesion is shown to play an important role in the epithelial to mesenchymal transition (EMT) and induction of an invasive phenotype, motility as well as regional and distant metastases $[3,4,15,16]$.

In the literature different mechanisms are discussed as involved in the repression of E-cadherin expression. Researchers suggest that knockdown of Rb gene and $\mathrm{NF}-\kappa \mathrm{B}$ protein activity resulted in reduced $\mathrm{CDH} 1$ expression $[17,18]$. Tumor invasion and metastasis are also regulated by the transcription family molecules, consisting of SNAIL and SLUG as well as ErbB-1/2 and ERK pathway members that suppress E-cadherin expression, leading to EMT $[15,19]$. Moreover, MAPK activation and Akt-induced EMT mediated through the activation of phosphatidylinositol 3 kinase (PIK3) are connected with downregulation of E-cadherin in many cancers [20, 21]. In CDH1 activity there can also be implicated tyrosine kinase-receptors such as Met, the hepatocyte growth factor (HGF) receptor [22]. The altered cell aggregation and increased motility in different cancers may be due to protein kinase D1 (PKD1) and E-cadherin interaction [23]. Expression of the $\mathrm{CDH} 1$ gene is also associated with up-regulation of MMP-9 as well as ZEB-1 and p63 protein expression [18, 24].

Methylation of $\mathrm{CpG}$ sites at the promoter region of the $\mathrm{E}$-cadherin gene played a key role in the inhibition of $\mathrm{CDH} 1$ expression and led to loss of tissue integrity and tumor progression [5, 25]. CpG island methylation in the promoter region of the $\mathrm{CDH} 1$ gene and consistent depression of the E-cadherin level contributes to the phenotypic heterogeneity and it is observed in primary tumors with different origin and correlates with tumor recurrences, nodal metastases and poor prognosis [26, 27]. What is of interest, reactive oxygen species 
(ROS) can also promote methylation of the promoter region of E-cadherin [28].

The purpose of this study was to elucidate E-cadherin gene expression at mRNA and protein levels as well as gene promoter DNA methylation status in cancer tissue to describe the correlations with clinicomorphological parameters in squamous cell laryngeal carcinoma.

\section{Material and methods}

Tissue samples, histological classification and morphological features

For this study 86 cases (84 men, 2 women; age 45-79 years; mean age $61.9 \pm 8$ years) of fresh tissue samples of surgically resected specimens from patients treated for squamous cell laryngeal carcinoma were utilized. The criteria for patient participation in this study were as follows:

- histologically confirmed diagnosis of carcinoma planoepitheliale,

- primary surgical resection of the larynx without receiving prior immuno-, radio- or chemotherapy,

- absence of distant metastases.

All specimens were confirmed by routine clinical diagnosis according to the UICC TNM classification of 2003 for head and neck carcinomas [29]. Morphologic estimation was performed on hematoxylin-eosin-stained (H\&E) sections in the peripheral parts of a tumor, according to the TFG classification [30]. We analyzed the following features in the most invasive, peripheral zones of the tumor: cytoplasmic differentiation, nuclear polymorphism, number of mitoses, mode of infiltration, depth of invasion and plasmalymphocytic infiltration of tumor front. These factors were assessed in at least five different regions of the peripheral part of the tumor (mag- nification 200x, number of mitoses magn. 400x). Each factor was graded according to a scale ranging from 1 to 4 . The total morphologic TFG score was computed as the sum of the 6 parameters, with a maximum score of 24 points. According to the TFG total score tumors were divided into 5 groups (ranks): 6-9, 10-13, 14-17, 18-21 and > 22 points of TFG. Clinicomorphological characteristics of the studied group are presented in Table 1. The investigations were performed with the approval of the Bioethical Commission of the Medical University of Lodz and the National Science Council, Poland (No. RNN/13/11/KE).

\section{Non-cancerous/cancerous epithelial cell isolation}

After radical/partial laryngectomy, the surgical tissue specimens were excised aseptically immediately after the operation from at least four tumor sites: two from the tumor margin and two sites of non-cancerous adjacent laryngeal epithelium (only after total laryngectomy due to ethical considerations). Fragments of tissues were washed with PBS for removal of contaminated blood and next inserted in RPMI 1640 medium (Biomed, Poland) supplemented with antibiotics streptomycin/penicillin/gentamycin 1\% v/v (Sigma, Aldrich, Germany). The whole procedure was performed on an ice plateau. Briefly, tissue specimens were cut with a surgical knife, and minced with a scalpel. This was done in RPMI 1640 medium (Biomed, Poland) supplemented with antibiotics streptomycin/penicillin/gentamycin 2\% v/v (Sigma, Aldrich, Germany). Fragments of tissues were then washed three times with Hanks solution (Biomed Lublin, Poland). Next the tumor and normal epithelial pieces were digested overnight (for 18 h) in Nunc Petri dishes with $0.16 \mathrm{mg} / \mathrm{ml}$ hyaluronidase (Sigma, Aldrich, Germany) and $0.55 \mathrm{mg} / \mathrm{ml}$ col-

Table 1. Clinicomorphological characteristics of group studied

\begin{tabular}{|lll}
\hline Feature & & $N(\%)$ \\
\hline Sex & men & $84(97.2)$ \\
& women & $2(2.8)$ \\
\hline Surgical treatment & partial laryngectomy & $23(26.7)$ \\
& total laryngectomy & $63(73.3)$ \\
& neck dissection & $46(53.5)$ \\
& selective neck dissection & $41(47.7)$ \\
\hline PT & radical neck dissection & $5(5.8)$ \\
\hline pN & pT1-pT2 & $29(33.7)$ \\
& pT3-pT4 & $57(69.3)$ \\
\hline G & pNo & $64(74.4)$ \\
histological differentiation & pN1-3 & $22(25.6)$ \\
& G1 & $10(11.6)$ \\
\hline Depth of invasion & G2 & $67(77.9)$ \\
& G3 & $9(10.5)$ \\
\hline Mode of invasion & CIS and invasion into lamina propria & $21(24.3)$ \\
& muscle tissue and cartilage invasion & $65(75.7)$ \\
\hline TFG & well-defined and less marked borderline & $33(38.5)$ \\
(tumor front grading) & no distinct borderline and diffuse growth & $53(61.5)$ \\
\hline
\end{tabular}


lagenase (Sigma, Aldrich, Germany) in the presence of the antibiotics streptomycin/penicillin/gentamycin $1 \% \mathrm{v} / \mathrm{v}$ (Sigma, Aldrich, Germany) at $37^{\circ} \mathrm{C}, 5 \% \mathrm{CO}_{2}$ (Cellstar Incubator). The digested tissues were pressed gently through a $50-\mu \mathrm{m}$ (mesh) sieve (Sigma, Aldrich, Germany) with RPMI 1640 medium (Biomed, Poland). Subsequently, the suspension was three times washed with PBS (without $\mathrm{Mg}^{2+}$ and $\mathrm{Ca}^{2+}$ ) for 20 min at $8^{\circ} \mathrm{C}$ by centrifugation in a MPW-350R centrifuge at 1800 $\mathrm{rpm} / 500 \mathrm{rcf}$ and poured over with dispase solution $2.4 \mathrm{U} / \mathrm{ml}$, incubated for $30 \mathrm{~min}$ in a $37^{\circ} \mathrm{C}$ and next resuspended in $1 \mathrm{ml}$ of PBS. The concentration of cells was estimated using a microscope and Bürker's chamber. To get rid of the apoptotic and necrotic cells the columns of Magnetic Cell Sorting Separator MACS (Miltenyi Biotec, Germany) and Dead Cell Removal Kit was used. The recovered cells were checked (typical epithelial morphology, immunohistochemical positive reactions with polyclonal antibodies [NCL-C11, Multi-Cytokeratin 4/5/6/8/10/13/18, RTU-D Novostatin Universal Detection Kit, NCL-L-DAB Liquid DAB Substrate Kit; Novocastra UK]) and counted for viability with the trypan blue staining method. The isolated laryngeal epithelial cells (tumor front cells and non-cancerous epithelial cells) were collected immediately after the procedure and RNAlater RNA Stabilization Reagent (QIAGEN GmbH, Hilden, Germany) was added to the cell pellets. Cell pellets were stored at $-80^{\circ} \mathrm{C}$ until isolation of RNA.

\section{Total RNA extraction and cDNA synthesis}

The total RNA was extracted using TRI Reagent (Sigma Aldrich, USA) according to the manufacturer's protocol. RNA was diluted in $20 \mu$ l of RNase-free water, quantified by spectrophotometry at $260 \mathrm{~nm}$ and stored at $-20^{\circ} \mathrm{C}$. RNA with a 260/280 nm ratio in the range 1.8-2.0 was considered high quality. First-strand cDNA was synthesized from each RNA pool using PCR Kit ver. 3.0 (Takara Bio Inc., Japan) according to the manufacturer's instructions. Briefly, $1 \mu \mathrm{g}$ of RNA was combined with 2.5 pmol of oligo dT-adapter primer, $4 \mu$ l of $25 \mathrm{mM} \mathrm{MgCl}_{2}$, $2 \mu$ l of $10 \times$ RNA PCR buffer, $2 \mu$ of 10 mM dNTP mixture, 20 units of RNase inhibitor, 5 units of AMV Reverse Transcriptase $\mathrm{XL}$, and RNase-free water to a total volume of $20 \mu \mathrm{l}$. The reaction took place at $42^{\circ} \mathrm{C}$ for $30 \mathrm{~min}$, followed by $95^{\circ} \mathrm{C}$ for $5 \mathrm{~min}$ and $5^{\circ} \mathrm{C}$ for $5 \mathrm{~min}$ in a GeneAmp PCR System 9700 (Perkin-Elmer Co, USA). cDNA was stored at $-20^{\circ} \mathrm{C}$.

\section{Real-time quantitative PCR (qRT-PCR)}

The real-time PCR was performed in a Mastercycler ep Realplex 4S (Eppendorf, Germany). Quantitative evaluation of $\mathrm{CDH} 1$ and HPRT1 (hypoxanthine phosphoribosyltransferase 1), as the control reference gene, was performed with commercially available TaqMan probes Hs01013959_m1 and Hs02800695_m1, respectively (Applied Biosystems, USA). PCR reactions were carried out in a total volume of $10 \mu \mathrm{l}$, containing $0.5 \mu \mathrm{l}$ of respective TaqMan probes, $3.5 \mu \mathrm{l}$ nuclease free water, $5 \mu$ l of universal master mix (Applied Biosystems, USA) and $1 \mu$ l of cDNA. The reactions were performed in duplicate. Abundance of studied genes' mRNA in samples was quantified by the $\Delta \mathrm{Ct}$ method. $\Delta \mathrm{Ct}$ (CtgeneCt HPRT1) values were recalculated into relative copy number values (number of copies of studied gene mRNA per 1000 copies of HPRT1 mRNA).

\section{Immunoblotting analysis}

Cytoplasmic and nuclear fractions were prepared separately from each sample by differential centrifugation of tissue homogenate in $0.25 \mathrm{M}$ sucrose in buffer containing $5 \mathrm{mM}$ $\mathrm{MgCl}_{2}, 0.5 \%$ Triton X-100, $1 \mathrm{mM}$ phenylmethylsulfonyl fluoride (PMSF), and $50 \mathrm{mM}$ Tris- $\mathrm{HCl}$ at $\mathrm{pH} 7.4$, in the presence of $10 \mathrm{mM}$ sodium molybdate. After centrifugation at $800 \times \mathrm{g}$ for $7 \mathrm{~min}$ the crude nuclear pellet was purified by centrifugation through $2.2 \mathrm{M}$ sucrose in the above buffer at 40000 $\times$ g for 60 min. Supernatant from the first spin, corresponding to the cytoplasmic fraction, was centrifuged at $1500 \times \mathrm{g}$ for $10 \mathrm{~min}$ to remove any remaining nuclei. Protein concentration in cellular fractions was estimated by means of the modified Lowry procedure [31] using bovine serum albumin as standard. Cytoplasmic and nuclear fraction proteins (50 $\mu \mathrm{g})$ were resolved on 8\% SDS-polyacrylamide slab gel [32] and electrotransferred onto Immobilon P membrane (Millipore Corp. Bedford, USA) using semi-dry technique [33]. After blocking in $0.5 \%$ BSA the membrane was incubated with anti-Ecadherin (sc-7870, H-108) antibody (Santa Cruz Biotechnology, USA). Following extensive washing with TBST buffer (Trisbuffered saline with Tween 20) the membrane was incubated with biotinylated mouse anti-rabbit lgG-HRP (sc-2357) antibody (Sigma Chemical Co., USA). Specificity of antigen-antibody interaction was tested by streptavidin/biotinylated horseradish peroxidase complex (Strept ABComplex/HRP) (Dako $A / S$, Denmark) and visualized with 4-chloro-1-naphthol and hydrogen peroxide as a substrate for HRP.

\section{Qualitative and quantitative estimations of immunoblots}

For qualitative and quantitative analysis of immunoblots, a video densitometer (Biotec-Fischer, Germany) and software Gel-Pro ${ }^{\circledR}$ Analyzer 3.0 (Media Cybernetics, USA) were used. The integrated optical density (IOD) of the bands, in a digitalized picture, was measured. For the immunoblot analysis the densitometric data of each sample were analyzed based on IOD, performed using a three-point scale corresponding to the densitometric ranges: no staining $(I O D=0)$, moderate staining $(I O D>0-0.4)$, strong staining $(I O D>0.4)$.

\section{Genomic DNA isolation and methyl-specific PCR (MSP)}

Genomic DNA was purified according to the Sambrook protocol [34]. Bisulfite treatment of genomic DNA was performed with the EZ DNA Methylation kit (ZYMO Research, Orange, CA, USA) according to the manufacturer's protocol. Modified DNA (50 ng) was used for each PCR with the following primers: methylated-specific primer set - sense 5'-GGTGAATTTTTAGTTAATTAGCCGGTAC-3' and antisense 5'-CATAACTAACCG AAAACGCCG-3', yielding a product of 204 bp; unmethylated-specific primer set - sense 5'-GGTAGGTGAATTTTTAGTTAATTAGTGGTA-3' and antisense 5'-ACCCATAACTAA CCAAA AACACCA-3', yielding a product of $211 \mathrm{bp}$. The PCR mixture $(50 \mu \mathrm{l}$ in total) contained $1 \times$ buffer with $1.5 \mathrm{mmol} / \mathrm{/} \mathrm{MgCl}_{2}, 0.2 \mathrm{mmol}$ dNTPs, $0.2 \mu \mathrm{mol}$ of each primer, and $4 \mu \mathrm{l}$ of DNA sample. PCR conditions were $10 \mathrm{~min}$ at $94^{\circ} \mathrm{C}$, after which $3 \mathrm{U}$ of Taq DNA polymerase was added, and $35 \mathrm{cycles}$ at $94^{\circ} \mathrm{C}$ for $50 \mathrm{~s}$, at $57^{\circ} \mathrm{C}$ for $40 \mathrm{~s}$, at $72^{\circ} \mathrm{C}$ 
for $90 \mathrm{~s}$, and a final extension at $72^{\circ} \mathrm{C}$ for 5 min. The positive control was performed on DNA from normal laryngeal tissue by using unmethylated-specific primers, and the negative control was prepared on PCR mixture without primers. The PCR products were migrated by electrophoresis on $2 \%$ agarose gel, with 100 bp DNA ladder as a DNA marker. As a positive and negative control we used Human Methylated \& Non-methylated DNA Set (Zymo Research).

\section{Statistical analysis of data}

Statistical analysis was performed using STATISTICA version 9.0 (StatSoft, Poland). $\Delta$ Ct values (which have a symmetrical theoretic distribution) obtained from quantitative real-time PCR were recalculated into relative copy number values. Obtained results were not normally distributed (Kolmogorov-Smirnov test) and therefore nonparametric statistical tests were used for analyzing the results (Mann-Whitney $U$ test and Kruskal-Wallis test). Categorical variables were compared by the chi-squared test. A value of $p<0.05$ was considered statistically significant.

\section{Results}

In the first stage, the expression pattern of the gene (CDH1g) as well as the protein nuclear (CDH1pn) and cytoplasmic fraction (CDH1pc) of E-cadherin in the tumor cells of laryngeal squamous cell carcinomas (SCLC) was studied. Our study confirmed the (CDH1g) mRNA positive expression in $88.4 \%(76 / 86)$ of all tumor samples and in $98.4 \%(62 / 63)$ of non-cancerous laryngeal epithelium cells. In these groups studied the mean values of E-cadherin mRNA were 1026.62 \pm 230.57 copies of $C D H 1$ mRNA per 1000 copies of HPRT1 mRNA and $1080.99 \pm 561.88$ copies of CDH1 mRNA per 1000 copies of HPRT1 mRNA, respectively. Significant differences in $\mathrm{CDH} 1 \mathrm{~g}$ expression between tumor marginal cells and noncancerous epithelial cells $(p=0.04)$ were observed. Positive protein nuclear fraction (CDH1pn) of E-cadherin expression in $69.8 \%(60 / 86)$ of cancerous cells and in $76.2 \%$ (48/63) of adjacent mucosa laryngeal cells were assessed. The mean values of CDH1pn level in cancerous and non-cancerous samples were $134.11 \pm 121.62$ IOD and $209.03 \pm 117.79$ IOD, respectively. Significant differences between $\mathrm{CDH1}$ 1p expression in tumor and adjacent laryngeal mucosa cells $(p=0.03)$ were found. A positive protein cytoplasmic fraction ( $\mathrm{CDH} 1 \mathrm{pc})$ of E-cadherin in $11.6 \%(10 / 86)$ of cancerous cells was observed. In no case of adjacent mucosa laryngeal cells was $\mathrm{CDH} 1$ c expression assessed. The mean value of CDH1pc level in cancerous samples was $49.96 \pm 124.07$ IOD. Moreover, the DNA methylation status of the CDH1 gene level, as an indi- cator of gene inactivation in this study, was estimated. E-cadherin methylation was observed in 49 of 86 tumor samples (56.9\%). In remaining tumor cells the negative status of $\mathrm{CDH} 1$ promoter methylation was noted. Subsequently, correlations were identified between the gene ( $\mathrm{CDH} 1 \mathrm{~g})$ and protein (CDH1p) of E-cadherin expression, the DNA methylation status of the gene levels and clinicopathological features, specifying the degree of carcinoma aggressiveness, to evaluate its possible role as a potential biomarker for tumor behavior in SCLC.

\section{The relationships between E-cadherin mRNA expression (CDH1g) and clinicopathological parameters in SCLC}

To investigate whether the CDH1 mRNA expression status can potentially determine clinicopathological features, the qRT-PCR results were juxtaposed with the pathological assessment of the primary tumor (pT status), the pathological assessment of the regional lymph nodes ( $\mathrm{pN}$ status), the histological grade $(\mathrm{G})$, the TFG total score and selected parameters of TFG classification. Representative examples of MSP-PCR analysis of tumor laryngeal cell samples are shown in Fig. 1. The most numerous group of laryngeal carcinomas was advanced tumors (pT3-pT4). In this group the mean values of E-cadherin mRNA were lower $(933.11 \pm 357.42$ copies of CDH1 mRNA per 1000 copies of HPRT1 mRNA) in comparison with less advanced carcinomas (pT1-pT2) characterized by higher mean values of CDH1 mRNA (1150.09 \pm 458.79 copies of $\mathrm{CDH} 1 \mathrm{mRNA}$ per 1000 copies of HPRT1 mRNA). The statistical analysis confirmed the presence of significant differences in the level of mRNA expression of CDH1 in tumor cells between pT1-pT2 and pT3-pT4 tumors $(p<0.001)$. Well-defined and less marked borderlines of tumor infiltration were characteristic for carcinomas with lower mean values of E-cadherin mRNA in positive tumor cells (985.22 \pm 295.09 copies of CDH1 mRNA per 1000 copies of HPRT1 mRNA). In contrast, diffuse growth and indistinct tumor front borderlines were most often seen in neoplasms characterized by higher mean values of CDH1 mRNA (1071.64 \pm 535.84 copies of CDH1 mRNA per 1000 copies of HPRT1 mRNA) in tumor cells. Statistical evaluation of the quantitative analysis results and the clinicomorphological parameters of laryngeal carcinomas showed that the expression of mRNA for the $\mathrm{CDH} 1$ in cells isolated from the peripheral part of the tumor was significantly different depending on the type of invasion ( $p=0.033$ ). The presence of a lower content of $\mathrm{CDH} 1 \mathrm{mRNA}$ in carcinoma cells was more frequent for tumors with more aggressive behavior determined by muscle tissue and car-

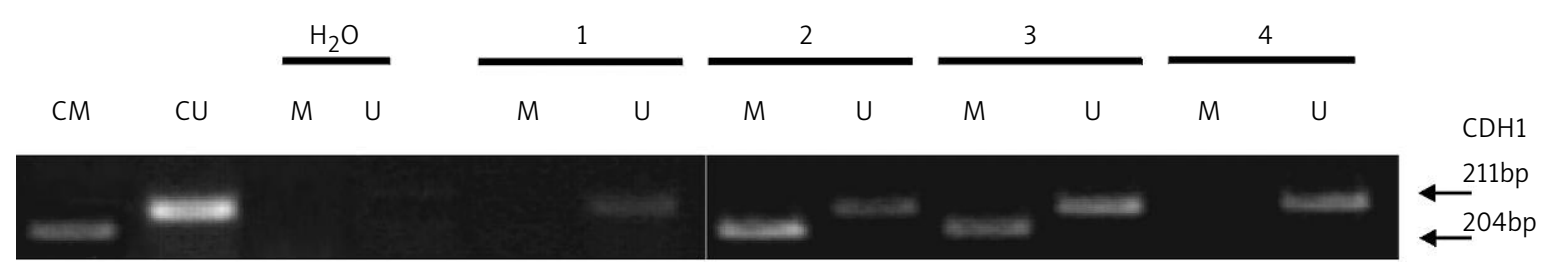

Fig. 1. Representative examples of MSP-PCR analysis of tumor laryngeal cell samples. Bisulfite-modified DNA was amplified with specific primers to detect methylated DNA (M) and unmethylated DNA (U); control for methylated genes (CM); control of unmethylated genes (CU); $\mathrm{H}_{2} \mathrm{O}$ : distilled water used as negative control in the PCR amplification 


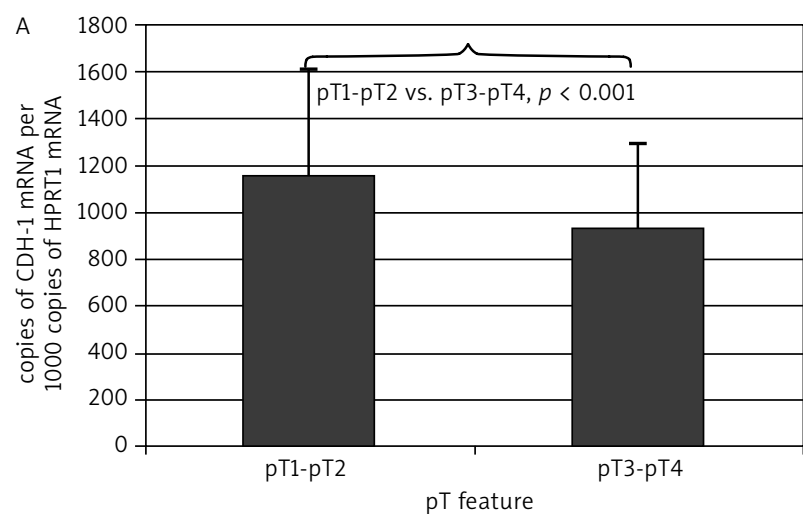

pT feature

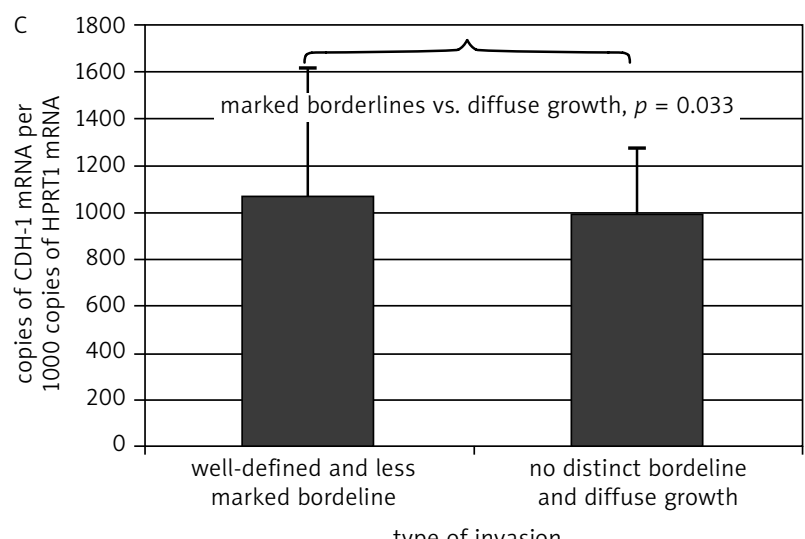

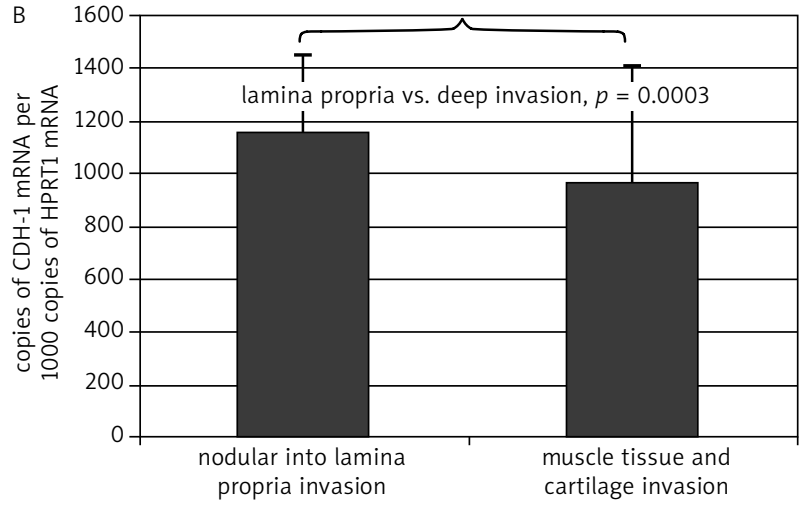

depth of invasion

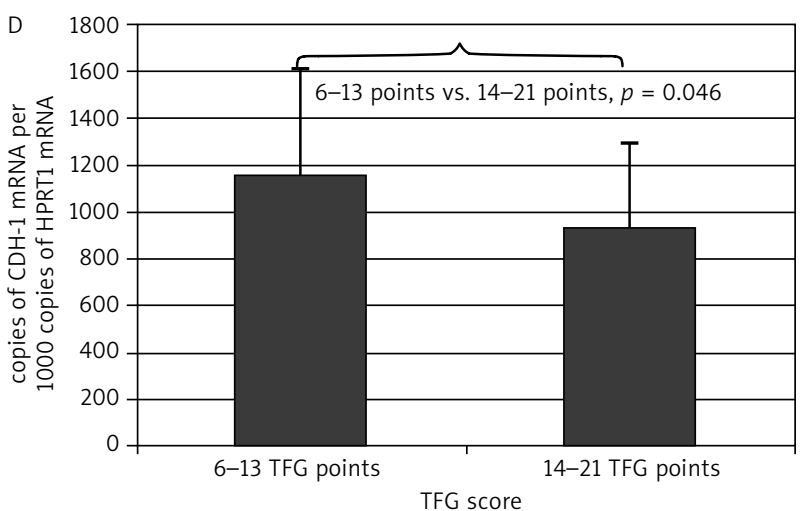

Fig. 2. Expression of E-cadherin mRNA depending on the clinicopathological characteristics in SCLC; $A)$ CDH1g mRNA expression in tumor laryngeal cells depending on pT feature $(p<0.001)$; B) the depth of invasion $(p=0.0003)$; C) the mode of invasion $(p=0.033)$; D) the tumor front grading total score $(p=0.046)$

tilage invasion ( $964.32 \pm 444.39$ copies of $\mathrm{CDH} 1$ mRNA per 1000 copies of HPRT1 mRNA) in comparison with less invasive carcinomas not exceeding the border of the lamina propria (1158.45 \pm 291.98 copies of CDH1 mRNA per 1000 copies of HPRT1 mRNA). The statistical analysis disclosed the presence of significant differences in the level of mRNA expression of E-cadherin in tumor cells between these groups ( $p$ < 0.001). Moreover, carcinomas with lower expression of $\mathrm{CDH} 1 \mathrm{mRNA}$ in tumor stroma cells were characterized by a higher total score of tumor front grading classification. In laryngeal carcinomas characterized by 14-21 points the mean values of E-cadherin mRNA were lower (1008.29 \pm 338.68 copies of CDH1 mRNA per 1000 copies of HPRT1 mRNA) in comparison with less advanced carcinomas with 6-13 points on the tumor front grading scale $(1043.65 \pm 518.35$ copies of CDH1 mRNA per 1000 copies of HPRT1 mRNA). Statistical analysis of $\mathrm{CDH} 1 \mathrm{mRNA}$ expression with respect to the degree of invasiveness of cancer lesions revealed that the average value of ribonucleic acid in cancer cells was significantly different according to TFG total score $(p=0.046)$. For other clinicopathological features, the $\mathrm{CDH} 1 \mathrm{~g}$ rate was uniform and independent of the intensity of these parameters. To sum up, the qRT-PCR results obtained for SCLC with more aggressive characteristics determined by a higher PT status, more disseminated tumor invasion and deep infiltration with muscle and cartilage involvement demonstrated a higher
$\mathrm{CDH} 1 \mathrm{~g}$ expression in tumor samples. The expression of E-cadherin mRNA depending on the clinicopathological characteristics in SCLC depending on $\mathrm{pT}$ feature, the depth of invasion, the mode of invasion and the tumor front grading total score is shown in Fig. 2.

\section{The relationships between E-cadherin protein (CDH1p) expression and clinicopathological parameters in SCLC}

To investigate whether the protein nuclear (CDH1pn) and cytoplasmic fraction (CDH1pc) of E-cadherin in the tumor cells of laryngeal squamous cell carcinomas can potentially determine clinicopathological features, the Western blot results were juxtaposed with the pathological assessment of the primary tumor (pT status), the pathological assessment of the regional lymph nodes (pN status), the histological grade $(\mathrm{G})$, the TFG total score and chosen parameters of the TFG classification. Representative results of E-cadherin protein expression analyses in homogenates of tumor laryngeal cells are shown in Fig. 3. The most advanced tumors (pT3-pT4) were characterized by lower mean values of E-cadherin nuclear protein level in tumor cells (101.15 \pm 110.90 IOD) in comparison with less advanced carcinomas (pT1-pT2) characterized by higher mean values of CDH1pn $(181.77 \pm 131.85$ IOD). The presence of significant differences in the level of CDH1pn expression in tumor cells between pT1-pT2 and pT3- 


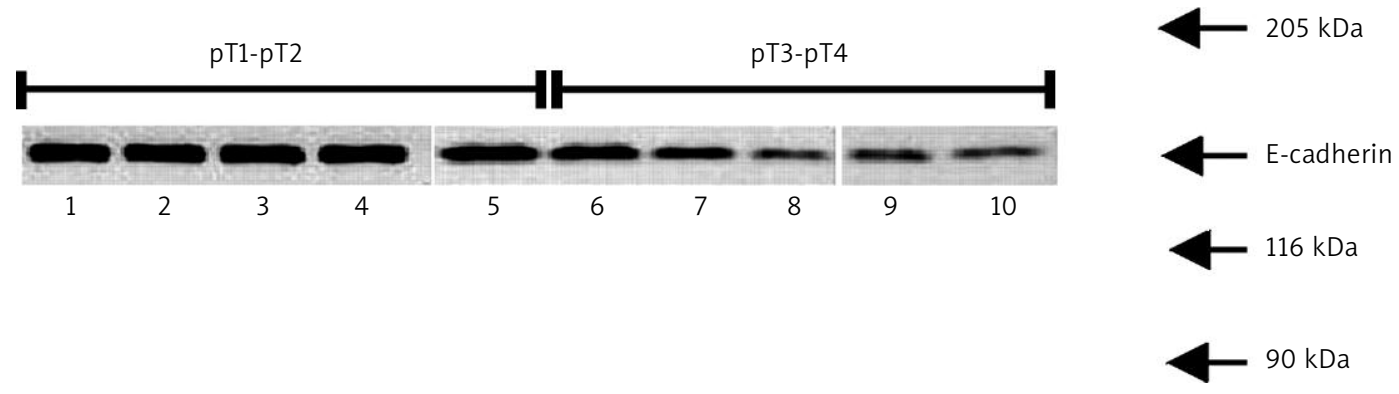

Fig. 3. Representative results of E-cadherin protein expression analyses in homogenates of tumor laryngeal cells. Lanes 1-5 $\rightarrow$ pT1-pT2 tumors and lanes $6-10 \rightarrow$ pT3-pT4 tumors. The electrophoretic mobilities of myosin (205 kDa), $\beta$-galactosidase (116 kDa) and fructose6 -phosphate kinase $(90 \mathrm{kDa})$ subunits, used as marker proteins running in a parallel lane, are indicated on the right

pT4 tumors ( $p=0.004$ ) was confirmed. The group of laryngeal carcinomas with well-defined and less marked borderlines of tumor invasion constituted cases with a higher average value of protein nuclear fraction (186.11 \pm 130.79 IOD). Tumors characterized by diffuse growth and indistinct tumor front borderlines were characterized by higher mean values of CDH1pn expression (96.01 \pm 107.91 IOD). The statistical analysis disclosed the presence of significant differences in the level of E-cadherin nuclear protein expression in tumor cells between these groups of tumors $(p=0.002)$. Moreover, the presence of the lower content of E-cadherin protein nuclear fraction in carcinoma cells was more frequent for tumors with more aggressiveness determined by muscle tissue and cartilage invasion $(105.71 \pm 118.61$ IOD) in comparison with less invasive tumors with borders to the lamina propria (201.63 \pm 117.72 IOD). The statistical analysis disclosed the presence of significant differences in the level of CDH1pn expression in tumor cells between these groups $(p=0.003)$. In addition, laryngeal carcinomas with $6-13$ points as well as tumors with 14-21 points of the total TFG score were described as SCLC with different levels of CDH1pn expression in tumor cells $(p=0.0002)$. In laryngeal carcinomas characterized by 14-21 points the mean values of CDH1pn were lower (95.55 \pm 109.07 IOD) in contrast to less advanced carcinomas with $6-13$ points (196.87 \pm 126.98 IOD). We found that the CDH1pn status had no significant correlations with other clinicomorphological parameters. Summing up, the Western blot results obtained for SCLC with more aggressive behavior characterized by a higher $\mathrm{pT}$ status, more disseminated tumor invasion and deep infiltration with muscle and cartilage involvement demonstrated lower CDH1p expression in tumor cells. The expression of E-cadherin protein level depending on the clinicopathological characteristics in SCLC depending on PT feature, the depth of invasion, the mode of invasion and the tumor front grading total score is shown in Fig. 4. Due to the positive protein cytoplasmic fraction $(\mathrm{CDH} 1 \mathrm{pc})$ of E-cadherin only in a small percentage of tumor cells (11.6\%) statistical analysis was not performed in the group studied. It should be noted, however, that all cases of SCLC with a positive level of $\mathrm{CDH} 1$ cytoplasmic fraction were characterized by less aggressive cancer lesions, determined by a lower pT status (pT1-pT2) and a lower total score (6-13 points) according to the tumor front grading classification.
The relationships of E-cadherin promoter methylation with (CDH1g) mRNA and E-cadherin protein (CDH1p) expression. The relationships between clinicopathological parameters in SCLC and E-cadherin promoter methylation

E-cadherin methylation was observed in 49 of 86 tumor samples (56.9\%). CDH1 mRNA expression was associated with promoter methylation status in the group studied. In laryngeal carcinomas with confirmed $\mathrm{CDH} 1$ methylation, mean values of E-cadherin mRNA were lower (628.56 \pm 523.95 copies of CDH1 mRNA per 1000 copies of HPRT1 mRNA) in comparison with tumors without methylated E-cadherin promoter (1178.56 \pm 406.90 copies of CDH1 mRNA per 1000 copies of HPRT1 mRNA). Downregulation of CDH1 mRNA expression was found to be statistically related to promoter methylation $(p<0.001)$. Nevertheless, our study did not disclose significant differences of E-cadherin protein (CDH1p) expression with promoter methylation status $(p=0.23)$. However, the occurrence of a tendency to a lower average value of protein nuclear fraction $(162.21 \pm 133.85$ IOD) in carcinomas with $\mathrm{CDH} 1$ methylation in contrast to tumors without promoter methylation (204.53 \pm 127.28 IOD) was observed. To investigate whether the E-cadherin promoter methylation status can potentially determine clinicopathological features, the methyl-specific PCR (MSP) results were juxtaposed with the pathological assessment of the primary tumor (pT status), the pathological assessment of the regional lymph nodes (pN status), the histological grade $(G)$, the TFG total score and selected parameters of TFG classification. E-cadherin promoter methylation in the group of advanced tumors (pT3-pT4) was noted in $52.6 \%$ (30/57) of cases in comparison with $24.1 \%$ $(7 / 29)$ in less advanced carcinomas (pT1-pT2). The statistical analysis confirmed the presence of significant differences in the level of promoter methylation in tumor cells between pT1-pT2 and PT3-pT4 tumors ( $p<0.0001)$. Similarly, the presence of a higher content of methylated $\mathrm{CDH} 1$ promoter in carcinoma cells was more frequent for tumors with more aggressive behavior determined by muscle tissue and cartilage invasion $(54.5 \%, 31 / 57)$ in comparison with less invasive carcinomas not exceeding the border of the lamina propria $(13.8 \%, 4 / 29)$. The statistical analysis disclosed the presence of significant differences in promoter methylation status in these groups $(p<0.01)$. We found that the CDH1 methylation status had no significant correlations with other 


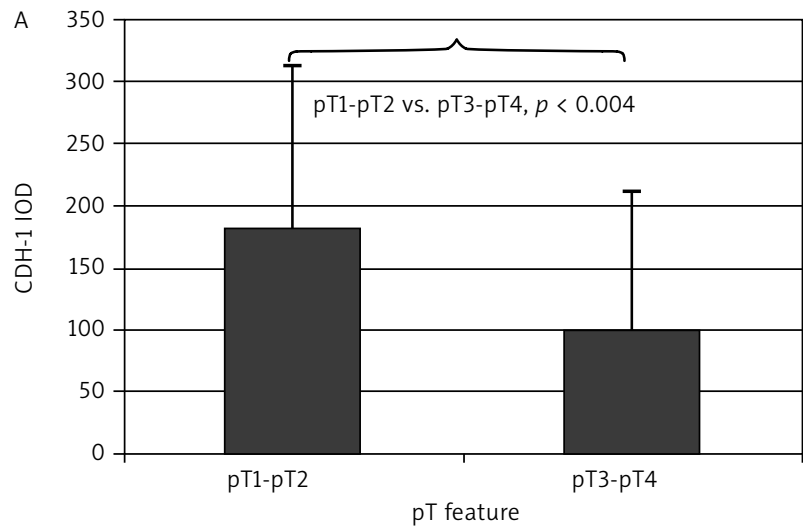

pT feature

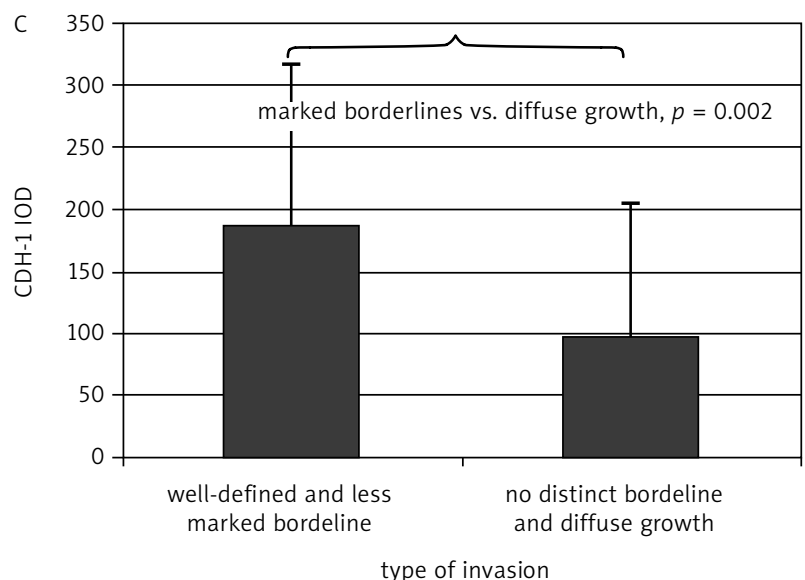

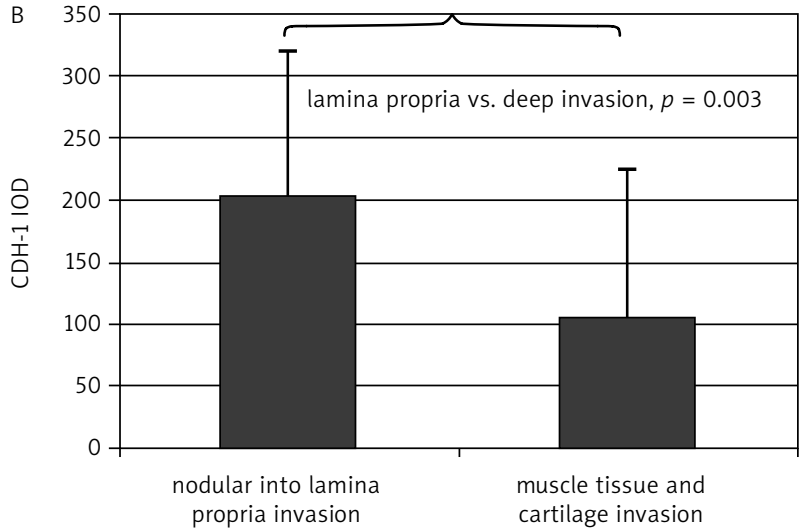

depth of invasion

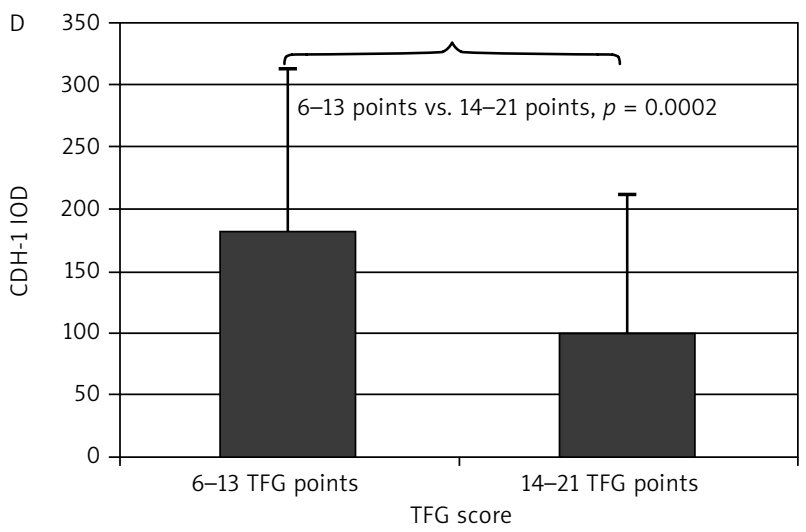

Fig. 4. Expression of E-cadherin protein level depending on the clinicopathological characteristics in SCLC; A) CDH1p expression in tumor laryngeal cells depending on pT feature $(p=0.004)$; B) the depth of invasion $(p=0.003)$; C) the mode of invasion $(p=0.002)$; D) the tumor front grading total score $(p=0.0002)$

clinicomorphological parameters. In summary, the MSP results obtained for SCLC with more aggressive behavior characterized by a higher pT status and deep infiltration with muscle and cartilage involvement demonstrated a higher frequently of methylated $\mathrm{CDH} 1$ promoter in tumor cells.

\section{Discussion}

E-cadherin $(\mathrm{CDH} 1)$ is a cell adhesion molecule that is downregulated in cancer tissues and is proposed as a useful phenotypic marker of tumor cell motility and an indicator of a higher degree of aggressiveness in tumors of different origin $[1-6,8,11-14,16,23,25,26]$. The study results suggest that $\mathrm{E}$-cadherin loss plays a role in progression and development of local and distant metastases, and its function assessment helps in the identification of patients with poor outcomes $[2,14,25]$. The high correlation between $\mathrm{CDH} 1$ inactivation status and the invasive and metastatic phenotype of tumor cells suggests crucial biological roles during tumorigenesis [1-6, 8, 11-14, 16, 23, 25, 26]. Epigenetic silencing is considered one of the leading mechanisms of E-cadherin impairment that converts this tumor suppressor into an oncogenic factor causing the acquisition of the aggressive behavior of tumor cells [1, 3-5, 25-28]. Nevertheless, the clinicopathological and prognostic value of E-cadherin in laryngeal carcinoma still remains unclear. Moreover, it should be emphasized that there seems to be a paucity of published data regarding the role of $\mathrm{CDH} 1$ status in the corresponding aggressiveness of the tumor in laryngeal carcinoma, estimated according to clinicopathological features. Therefore, study into the gene and protein $\mathrm{CDH} 1$ expression of cancer tissues and the DNA methylation status of the gene levels was undertaken to determine their role as potential biomarkers of tumor behavior in laryngeal carcinoma. The results confirm high positive expression of the mRNA and protein nuclear fraction of E-cadherin in laryngeal tissues, either in adjacent mucosa cells or, to a lesser extent, in neoplastic cells. These data are in agreement with the findings of studies reporting a wide dispersion of $\mathrm{CDH} 1$ activity rates in different carcinomas. In some studies, positive expression of E-cadherin was found in $33-86.5 \%$ of various types of human HNSCC, including $39.3-72 \%$ of cases of CDH1 methylation $[2,5,6,11,12,21,25,26,28]$. A wide range of results for $\mathrm{E}$-cadherin expression in neoplastic tissues may stem from the different (either mRNA or protein) fractions and methods used in these studies and the predominance of cases with different pathological extents of the primary tumors. Moreover, the degree of invasiveness and grade of primary tumors can be crucial in the obtained results. Specifically, grade 1 carcinomas of different origin in comparison with more advanced tumors, characterized by grade 2 and grade 3 , showed preserved $\mathrm{E}$-cadherin expression in the range of $40-100 \%$ [11]. We provide the first evidence that the gene 
and protein E-cadherin expression and the DNA methylation status of the gene levels play an essential role in determining the clinicomorphological features of laryngeal cancer cells. Significant relationships have been demonstrated between the gene and protein $\mathrm{CDH} 1$ expression and clinicomorphological features according to tumor front grading, which is a useful method for determination of neoplastic growth dynamics in the peripheral infiltration edge. In addition, the differences between E-cadherin mRNA expression as well as $\mathrm{CDH} 1$ protein nuclear fraction and the aggressive potential of the laryngeal carcinomas, estimated according to clinicomorphological features, such as the pathological assessment of the primary tumor (pT), mode of invasion and the type of infiltration and tumor front grading score, have also been disclosed. Specifically, laryngeal tumors characterized by a higher pT status (classified as PT3-pT4), more disseminated growth (diffuse invasion or no distinct borderlines in the tumor front) as well as more aggressive behavior of neoplastic infiltration (muscle tissue and cartilage invasion), an indicator of local weaker cell-cell adhesions, demonstrated lower $\mathrm{CDH} 1$ expression in tumor samples. The importance of E-cadherin mRNA and $\mathrm{CDH} 1$ protein staining was also indicated to be a reliable potential biomarker of tumor progression estimated on the basis of the tumor front grading score. Laryngeal carcinomas with lower aggressive behavior determined by a smaller number of TFG total points (6-13 points) were found to demonstrate a higher E-cadherin gene and protein content in tumor samples. Other studies that have investigated $\mathrm{CDH} 1$ content in both primary tumors and corresponding metastatic lesions also suggest that E-cadherin activity plays an important role in tumor pathogenesis and the determination of growth and progression [2, 12, 14, 25]. For instance, Tang et al. [2] revealed the relationship of E-cadherin expression with node status and overall survival as well as recurrence status in breast cancer. The authors reported that $\mathrm{CDH1}$-negative patients experienced shorter disease-free interval, poorer overall survival and higher frequency of recurrence or metastasis than E-cadherinpositive patients. Batistatou et al. [12] disclosed the association between E-cadherin expression and the development and progression of colorectal carcinoma. An E-cadherinnegative expression profile was associated with lymph node metastases and $81 \%$ of metastatic tumors were positive for $\mathrm{CDH} 1$ in colorectal tumors studied [12]. Moreover, reduced or lost E-cadherin expression was also significantly associated with widely invasive growth, insular morphology and a lower degree of differentiation in investigations on thyroid carcinomas by Brecelj et al. [13]. However, no statistical significance in $\mathrm{CDH} 1$ expression with regard to other clinicopathological parameters, i.e. patient sex and age or tumor size, were disclosed in these studies. Interestingly, Rakha et al. [14] did not confirm differences in the CDH1 content distribution in relation to parameters such as lymph node status; however, the E-cadherin expression and a reduced disease-free interval and overall survival and also indicators of poor prognosis including larger tumor size, higher histological grade, and development of distant metastasis in univariate analysis were found to be significant in invasive breast cancers. Chang et al. [25] also confirmed that underexpres- sion had adverse effects on the prognosis of patients who were treated by primary surgery due to oral tongue carcinoma. The authors found positive higher $\mathrm{CDH} 1$ immunohistochemical staining of tumor tissues, recurrent tumors and nodal metastases. Goyal et al. [8] noted no significant difference in the expression of E-cadherin between the various TNM stages in breast carcinomas. Moreover, the molecular marker did not significantly influence survival. In our opinion, discrepancies in the results reported on the relationships between E-cadherin expression and tumor-related clinicomorphological parameters may stem from different pathomorphological methods and the heterogeneous group of patients with different tumor localization as well as the number of patients included in the studies. It was also noted that the methylated $\mathrm{CDH} 1$ promoter status in tumor cells is a good indicator of tumor aggressiveness of neoplastic laryngeal tissues. Significant differences between laryngeal carcinomas with and without methylation of $\mathrm{CpG}$ sites at the promoter region of E-cadherin as well as the aggressive tumor potential, according to clinicomorphological features, such as the pathological assessment of the primary tumor (pT), mode of invasion and the type of infiltration and tumor front grading score were disclosed. Specifically, more invasive laryngeal tumors characterized by a higher pT status (classified as pT3-pT4) and more aggressive behavior of neoplastic infiltration (muscle tissue and cartilage invasion) demonstrated a higher frequency of DNA methylation status of the $\mathrm{CDH} 1$ gene in the tumor sample. These results are in line with other reports which indicate that methylated E-cadherin promoter status is a potent parameter implicated in cancer development and prognosis [1-6, 8, 11-14, 16, 23, 25, 26]. In the literature the researchers often introduce the clinicomorphological implications of E-cadherin promoter methylation $[1,5,8,25,26,28]$. For example, Chang et al. [8] observed that promoter methylation observed in primary oral tongue tumors reflects a more aggressive phenotype according to clinicomorphological features, such as lymph node metastases and recurrences. Caldeira et al. [5] also suggested that abnormal $\mathrm{CDH} 1$ methylation occurs with high frequency in infiltrating breast cancers associated with a decrease in E-cadherin expression. It was noted in a subgroup of cases characterized by loss of expression of $\mathrm{CDH} 1$ and other important genes for the mammary carcinogenesis process, probably due to the disruption of the mechanism of maintenance of DNA methylation in tumor cells. Interestingly, other researchers also found that E-cadherin promoter methylation observed in primary tumors tends to be more frequent in lymph-node positive cases and recurrent tumors but without connections with other clinicopathological features. All these findings suggest that E-cadherin promoter methylated tumor cells show a higher migration capacity and this determines the acquisition of the invasive and metastatic phenotype of cancers of various origin.

This work was supported by grants from the National Science Council, Poland (N403 043 32/2326).

The authors declare no conflict of interest. 


\section{References}

1. van Horssen R, Hollestelle A, Rens JA, Eggermont AM, Schutte M, Ten Hagen TL. E-cadherin promotor methylation and mutation are inversely related to motility capacity of breast cancer cells. Breast Cancer Res Treat 2012; 136: 365-77.

2. Tang D, Xu S, Zhang Q, Zhao W. The expression and clinical significance of the androgen receptor and E-cadherin in triple-negative breast cancer. Med Oncol 2012; 29: 526-33.

3. Catalano MG, Fortunati N, Pugliese M, et al. Histone deacetylase inhibition modulates E-cadherin expression and suppresses migration and invasion of anaplastic thyroid cancer cells. J Clin Endocrinol Metab 2012; 97: E1150-9.

4. von Burstin J, Eser S, Paul MC, et al. E-cadherin regulates metastasis of pancreatic cancer in vivo and is suppressed by a SNAIL/HDAC1/HDAC2 repressor complex. Gastroenterology 2009; 137: 361-71.

5. Caldeira JR, Prando EC, Quevedo FC, Neto FA, Rainho CA, Rogatto SR. $\mathrm{CDH} 1$ promoter hypermethylation and E-cadherin protein expression in infiltrating breast cancer. BMC Cancer 2006; 6: 48

6. Bukholm IR, Nesland JM, Bukholm G. Expression of adhesion proteins E-cadherin, alpha-catenin, beta-catenin and gamma-catenin is different in T1 and T2 breast tumours. Pathology 2006; 38: 403-7.

7. Thévenod F, Chakraborty PK. The role of Wnt/beta-catenin signaling in renal carcinogenesis: lessons from cadmium toxicity studies. Curr Mol Med 2010; 10: 387-404.

8. Goyal A, Martin TA, Mansel RE, Jiang WG. Real time PCR analyses of expression of E-cadherin, alpha-, beta- and gamma-catenin in human breast cancer for predicting clinical outcome. World J Surg Oncol 2008; 6: 56.

9. Brembeck FH, Rosário M, Birchmeier W. Balancing cell adhesion and Wnt signaling, the key role of beta-catenin. Curr Opin Genet Dev 2006; 16: 51-9.

10. David JM, Rajasekaran AK. Dishonorable discharge: the oncogenic roles of cleaved E-cadherin fragments. Cancer Res 2012; 72: 2917-23.

11. Batistatou A, Peschos D, Tsanou H, Charalabopoulos A, Nakanishi Y, Hirohashi S, Agnantis NJ, Charalabopoulos K. In breast carcinoma dysadherin expression is correlated with invasiveness but not with E-cadherin. Br J Cancer 2007; 96: 1404-08.

12. Batistatou A, Charalabopoulos AK, Scopa CD, Nakanishi Y, Kappas A, Hirohashi S, Agnantis NJ, Charalabopoulos K. Expression patterns of dysadherin and E-cadherin in lymph node metastases of colorectal carcinoma. Virchows Arch 2006; 448: 763-67.

13. Brecelj E, Frković Grazio S, Auersperg M, Bracko M. Prognostic value of E-cadherin expression in thyroid follicular carcinoma. Eur J Surg Oncol 2005; 31: 544-48.

14. Rakha EA, Abd El Rehim D, Pinder SE, Lewis SA, Ellis IO. E-cadherin expression in invasive non-lobular carcinoma of the breast and its prognostic significance. Histopathology 2005; 46: 685-93.

15. Gallo D, Ferlini C, Scambia G. The epithelial-mesenchymal transition and the estrogen-signaling in ovarian cancer. Curr Drug Targets 2010; 11: 474-81.

16. Wells A, Yates C, Shepard CR. E-cadherin as an indicator of mesenchymal to epithelial reverting transitions during the metastatic seeding of disseminated carcinomas. Clin Exp Metastasis 2008; 25: 621-8.

17. Arima Y, Inoue Y, Shibata T, Hayashi H, Nagano O, Saya H, Taya Y. Rb depletion results in deregulation of $\mathrm{E}$-cadherin and induction of cellular phenotypic changes that are characteristic of the epithelialto-mesenchymal transition. Cancer Res 2008; 68: 5104-12.

18. Chua HL, Bhat-Nakshatri P, Clare SE, Morimiya A, Badve S, Nakshatri H. NF-kappaB represses E-cadherin expression and enhances epithelial to mesenchymal transition of mammary epithelial cells: potential involvement of ZEB-1 and ZEB-2. Oncogene 2007; 26: 711-24.

19. Conacci-Sorrell M, Simcha I, Ben-Yedidia T, Blechman J, Savagner P, Ben-Ze'ev A. Autoregulation of E-cadherin expression by cadherin cadherin interactions: the roles of beta-catenin signaling, Slug, and MAPK. J Cell Biol 2003; 163: 847-57.

20. Hong KO, Kim JH, Hong JS, Yoon HJ, Lee JI, Hong SP, Hong SD. Inhibition of Akt activity induces the mesenchymal-to-epithelial reverting transition with restoring $\mathrm{E}$-cadherin expression in $\mathrm{KB}$ and KOSCC-25B oral squamous cell carcinoma cells. J Exp Clin Cancer Res 2009; 28: 28-31.
21. Reddy P, Liu L, Ren C, et al. Formation of E-cadherin-mediated cellcell adhesion activates AKT and mitogen activated protein kinase via phosphatidylinositol 3 kinase and ligand-independent activation of epidermal growth factor receptor in ovarian cancer cells. Mol Endocrinol 2005; 19: 2564-78.

22. Matteucci E, Ridolfi E, Desiderio MA. Hepatocyte growth factor differently influences Met-E-cadherin phosphorylation and downstream signaling pathway in two models of breast cells. Cell Mol Life Sci 2006; 63: 2016-26.

23. Syed V, Mak P, Du C, Balaji KC. Beta-catenin mediates alteration in cell proliferation, motility and invasion of prostate cancer cells by differential expression of E-cadherin and protein kinase D1. J Cell Biochem 2008; 104: 82-95.

24. Slaton JW, Karashima T, Perrotte P et al. Treatment with low-dose interferon-alpha restores the balance between matrix metalloproteinase- 9 and $\mathrm{E}$-cadherin expression in human transitional cell carcinoma of the bladder. Clin Cancer Res 2001; 7: 2840-53.

25. Chang HW, Chow V, Lam KY, Wei WI, Yuen A. Loss of E-cadherin expression resulting from promoter hypermethylation in oral tongue carcinoma and its prognostic significance. Cancer 2002; 94 : 386-92.

26. Jensen K, Patel A, Hoperia V, Larin A, Bauer A, Vasko V. Dynamic changes in E-cadherin gene promoter methylation during metastatic progression in papillary thyroid cancer. Exp Ther Med 2010; 1: 457-62.

27. Zou D, Yoon HS, Perez D, Weeks RJ, Guilford P, Humar B. Epigenetic silencing in non-neoplastic epithelia identifies E-cadherin (CDH1) as a target for chemoprevention of lobular neoplasia. J Pathol 2009; 218: 265-72.

28. Lim SO, Gu JM, Kim MS, Kim HS, Park YN, Park CK, Cho JW, Park YM, Jung $\mathrm{G}$. Epigenetic changes induced by reactive oxygen species in hepatocellular carcinoma: methylation of the E-cadherin promoter. Gastroenterology 2008; 135: 2128-40.

29. O'Sullivan B, Shah J. New TNM staging criteria for head and neck tumors. Semin Surg Oncol 2003; 21: 30-42.

30. Starska K, Kulig A, Łukomski M. Tumor front grading in prediction of survival and lymph node metastases in patients with laryngeal carcinoma. Adv Med Sci 2006; 51: 200-4.

31. Cadman E, Bostwick JR, Eichberg J. Determination of protein by a modified Lowry procedure in the presence of some commonly used detergents. Anal Biochem 1979; 96: 21-3.

32. Laemmli UK. Cleavage of structural proteins during the assembly of the head of bacteriophage T4. Nature 1970; 227: 680-5.

33. Jacobson G, Karsnas P. Important parameters in semi-dry electrophoretic transfer. Electrophoresis 1990; 11: 46-52.

34. Sambrook J, Fritsch EF, Maniatis T. Molecular cloning: a laboratory manual. Cold Spring Harbor, New York 1989; 181-85.

\section{Address for correspondence}

\section{Katarzyna Starska}

$1^{\text {st }}$ Chair and Department of Otholaryngology

Medical University of Lodz

Kopcińskiego 22

90-150 Łódź, Poland

e-mail: katarzyna.starska@umed.lodz.pl

Submitted: $\quad 28.10 .2012$

Accepted: $\quad$ 8.05.2013 